\title{
THRUST POROUS BEARING WITH ROUGH SURFACES LUBRICATED BY A ROTEM-SHINNAR FLUID
}

\author{
Anna WALICKA*, Edward WALICKI* \\ *Faculty of Mechanical Engineering, ul. Szafrana 2, P.O.Box 47, University of Zielona Góra, 65-516 Zielona Góra, Poland \\ A.Walicka@ijame.uz.zgora.pl, E.Walicki@ijame.uz.zgora.pl
}

received 13 June 2014, revised 10 February 2016, accepted 23 February 2016

\begin{abstract}
In the paper the influence of both bearing surfaces roughness and porosity of one bearing surface on the pressure distribution and load-carrying capacity of a thrust bearing surfaces is discussed. The equations of motion of a pseudo-plastic fluid of Rotem-Shinnar, are used to derive the Reynolds equation. After general considerations on the flow in a bearing clearance and in a porous layer using the Morgan-Cameron approximation and Christensen theory of hydrodynamic lubrication the modified Reynolds equation is obtained. The analytical solutions of this equation for the cases of squeeze film bearing and externally pressurized bearing are presented. As a result one obtains the formulae expressing pressure distribution and load-carrying capacity. Thrust radial bearing with squeezed film is considered as a numerical example.
\end{abstract}

Key words: Pseudo-Plastic Fluid, Rotem-Shinnar Model, Thrust Bearing, Porous Layer, Christensen Roughness

\section{INTRODUCTION}

Viscosity of lubricating oils decreases with an increase of temperature. This viscosity increases with the additives concentration and it is relatively independent on temperature and usually exhibits a non-linear relation between the shear stress and the rate of shear in shear flow. There is no generally acceptable theory taking into account the flow behavior of non-Newtonian lubricants. Studies have been done on fluid film lubrication employing several models such as micropolar (see e.g.: Walicka, 1994), couple-stress (Walicki and Walicka, 1998), mixture (Khonsari and Dai, 1992), viscoplastic (Lipscomb and Denn, 1984; Dorier and Tichy, 1992), pseudo-plastic (Wada and Hayashi, 1971; Swamy et al., 1975; Rajalingam et al., 1978). Naturally, this list is not complete and given only to present possibility of mathematical modeling. More complete list may be found in (Walicka, 2002; Walicki, 2005).

In recent years, a considerable amount of tribology research has been devoted to the study of the effect of surface roughness or geometric imperfections on hydrodynamic lubrication because the bearings surfaces, in practice, are all rough and the height of the roughness asperities may have the same order as the mean bearing clearance. Under these conditions, the surface roughness affects the bearing performance considerably.

The work in this area has mainly been confined to impermeable surfaces. The well-established stochastic theory of hydrodynamic lubrication of rough surfaces developed by Christensen (1970) formed the basis of this paper. In a series of works (Bujurke et al., 2007; Lin, 2000; 2001; Prakash and Tiwari, 1985; Walicka 2009; 2012; Walicka and Walicki, 2002a; 2002b) the model was applied to the study of the surface roughness of various geometrical configurations.

Porous bearings have been widely used in industry for a long time. Basing on the Darcy model, Morgan and Cameron (1957) first presented theoretical research on these bearings. To get a better insight into the effect of surface roughness in porous bearings, Prakash and Tiwari (1984) developed a stochastic theory of hydrodynamic lubrication of rough surfaces proposed by Christensen (1970).

The modified Reynolds equation (Gurujan and Prakash, 1999) applicable to two types of directional roughness structure were used by Walicka and Walicki (2002a; 2002b) to find bearing parameters for the squeeze film between two curvilinear surface.

In this paper the Rotem-Shinnar fluid model is used to describe the pseudo-plastic behaviour of a lubricant. The modified Reynolds equation is derived and its solution for the curvilinear thrust bearing is presented. The analysis is based on the assumption that the porous matrix consists of a system of capillaries of very small radii which allows a generalization of the Darcy law and use of the Morgan-Cameron approximation for the flow in a porous layer. According to Christensen's stochastic model (1970), different forms of Reynolds equations are derived to take account of various types of surface roughness. Analytical solutions for the film pressure are presented for the longitudinal and circumferential roughness patterns.

\section{DERIVATION OF THE REYNOLDS EQUATION FOR A ROTEM-SHINNAR FLUID}

It may be assumed that lubricating oils, with a viscosity index improver added, exhibit the same characteristics as pseudoplastic fluids. Rotem and Shinnar (1961) proposed a method for expressing empirically the relation between the stress and the shear rate as

$\frac{d \gamma}{d t}=\frac{\tau}{\mu}\left(1+\sum_{i=-1}^{n} k_{i} \tau^{2 i}\right)$. 
Retaining only the first order term $(i=1)$ the above equation reduces to

$\mu \frac{d \gamma}{d t}=\tau+k \tau^{3}$.

Typical flow curves are shown in Fig.1. Since $\mu$ is the tangent at the original point of the flow curves, shown in Fig.1, $\mu$ is the initial viscosity. If the values of $\mu$ do not vary, the non-linearity of the flow curve increases with the value of $k$, which means the coefficient of pseudo-plasticity. In pseudo-plastic fluids $k \neq 0$ and in Newtonian fluids $k=0$.

Therefore, in Newtonian fluids, the initial viscosity becomes the viscosity given by Newton's law.

The three-dimensional notation of Eq.(2) may be expressed as (Walicka, 2002)

$$
\mu \boldsymbol{A}_{1}=\boldsymbol{\Lambda}\left(1+k \Lambda^{2}\right) \quad \text { where } \quad \Lambda=\left[\frac{1}{2} \operatorname{tr}\left(\boldsymbol{\Lambda}^{2}\right)\right]^{\frac{1}{2}}
$$

is the magnitude of the second-order shear stress tensor $\boldsymbol{\Lambda}$, but $\boldsymbol{A}_{1}$ is the first Rivlin-Ericksen kinematic tensor.

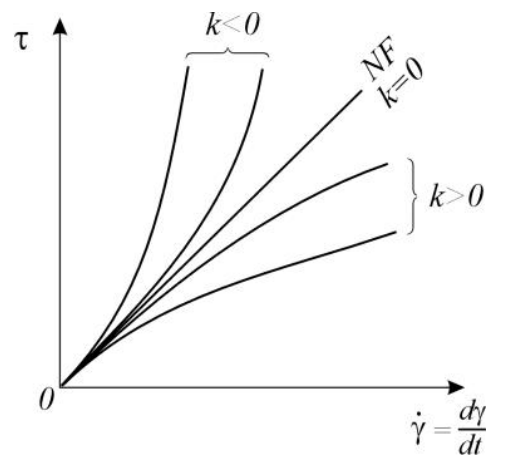

Fig. 1. Flows curves of a Rotem-Shinnar fluid of the first order $(i=1)$; symbol NF means a Newtonian fluid

Let us consider a thrust bearing with a curvilinear profile of the working surfaces shown in Fig.2. The upper bound of a porous layer is described by the function $R(x)$ which denotes the radius of this bound. The nominal bearing clearance thickness is given by the function $h(x, t)$, while the porous layer thickness is given by $H_{p}=$ const.

The expression for the film thickness is considered to be made up of two parts.

$H=h(x, t)+h_{s}(x, \vartheta, \xi)$,

where: $h(x, t)$ represents the nominal smooth part of the film geometry, while $h_{s}=\delta_{r}+\delta_{s}$ denotes the random part resulting from the surface roughness asperities measured from the nominal level, $\xi$ describes a random variable which characterizes the definite roughness arrangement. An intrinsic curvilinear orthogonal coordinate system $x, \vartheta, y$ linked with the upper surface of a porous layer is also presented in Fig. 2 .

Taking into account the considerations of the works (Walicka, 2002; Walicki, 2005) one may present the equation of continuity and the equations of motion of a Rotem-Shinnar fluid for axial symmetry in the form

$\frac{1}{R} \frac{\partial\left(R v_{x}\right)}{\partial x}+\frac{\partial v_{y}}{\partial y}=0$,

$\frac{\partial \Lambda_{x y}}{\partial y}=\frac{\partial p}{\partial x}, \quad \frac{\partial p}{\partial y}=0$. a)

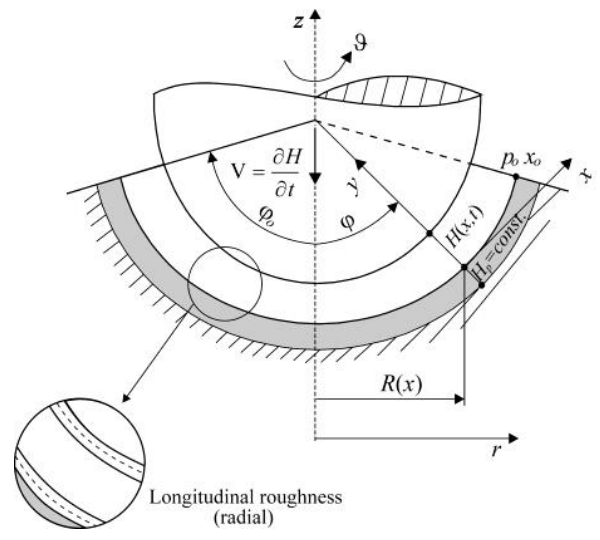

b)

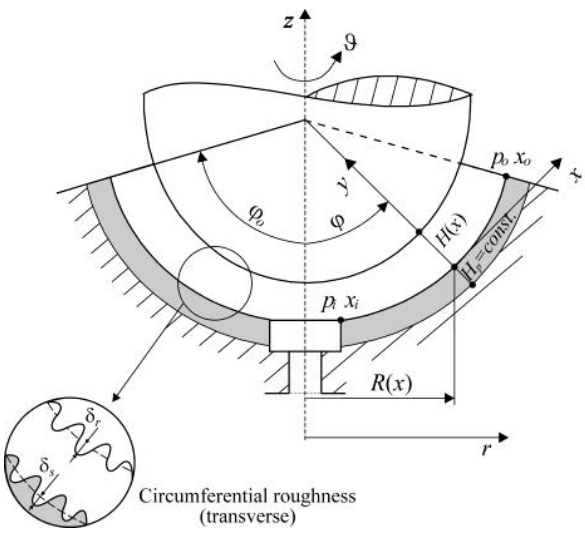

Fig. 2. Geometry of a curvilinear thrust bearing; (a) squeeze film bearing, (b) externally pressurized bearing

The constitutive equation (3) takes the form:

$\mu \frac{\partial v_{x}}{\partial y}=\Lambda_{x y}+k \Lambda_{x y}^{3}$.

The problem statement is complete after specification of boundary conditions. These conditions for velocity component are stated as follows:

$v_{x}(x, 0, t)=0, \quad v_{x}(x, H, t)=0$,

$v_{y}(x, 0, t)=V_{H}, \quad v_{y}(x, H, t)=\frac{\partial H}{\partial t}=\dot{H}$.

Solving the equations of motion (5), (6) and the constitutive taking into account equation (7) one obtains the Reynolds equation [detailed solution may be found in works (Walicka, 2002; Walicki, 2005)]

$\frac{1}{R} \frac{\partial}{\partial x} R H^{3}\left[\frac{\partial p}{\partial x}+\frac{3}{20} k H^{2}\left(\frac{\partial p}{\partial x}\right)^{3}\right]=12 \mu\left(\frac{\partial H}{\partial t}-V_{H}\right)$

for a lubricating pseudo-plastic fluid of Rotem-Shinnar. If $k=0$, the above equation is identical to the Reynolds equation for Newtonian lubricant (Walicki, 1977).

\section{MODIFIED REYNOLDS EQUATION FOR A BEARING WITH A POROUS PAD}

To solve Eq.(10) let us study the flow of a Rotem-Shinnar fluid in the porous layer. Assume that this layer consists a system of capillaries with an average radius $\mathrm{r}_{\mathrm{c}}$ and porosity $\phi_{p}$. Let the porous layer be homogeneous and isotropic and let the flow within the layer satisfy the modified Darcy's law. Thus one has (Walicki, 2005): 
$\bar{v}_{x}=\frac{\Phi_{p}}{\mu}\left(-\frac{\partial \bar{p}}{\partial x}\right)+\frac{\Phi_{p}}{\mu} \frac{k r_{c}^{2}}{6}\left(-\frac{\partial \bar{p}}{\partial x}\right)^{3}$

$\bar{v}_{y}=\frac{\Phi_{p}}{\mu}\left(-\frac{\partial \bar{p}}{\partial y}\right)+\frac{\Phi_{p}}{\mu} \frac{k r_{c}^{2}}{6}\left(-\frac{\partial \bar{p}}{\partial y}\right)^{3}$,

where: $\bar{v}_{x}, \bar{v}_{y}$ are velocity components in the porous layer and

$\Phi_{p}=\frac{\phi_{p} r_{c}^{2}}{8}$

is a permeability of the porous layer but $\phi_{p}$ is a coefficient of porosity.

Since the cross velocity component $\bar{v}_{y}$ must be continuous at the porous wall-fluid film interface and must be equal to $V_{H}$, we have then - by virtue of Eqs (10) and (11) - the following form of the modified Reynolds equation

$\frac{1}{R} \frac{\partial}{\partial x} R H^{3}\left[\frac{\partial p}{\partial x}+\frac{3}{20} k H^{2}\left(\frac{\partial p}{\partial x}\right)^{3}\right]=$

$=12 \mu\left[\frac{\partial H}{\partial t}-\left.\frac{\Phi_{p}}{\mu}\left\{\left(-\frac{\partial \bar{p}}{\partial y}\right)+\frac{k r_{c}^{2}}{6}\left(-\frac{\partial \bar{p}}{\partial y}\right)^{3}\right\}\right|_{y=0}\right]$.

Using the Morgan-Cameron approximation (Morgan and Cameron, 1957) one obtains

$\left.\left\{\left(-\frac{\partial \bar{p}}{\partial y}\right)+\frac{k r_{c}^{2}}{6}\left(-\frac{\partial \bar{p}}{\partial y}\right)^{3}\right\}\right|_{y=0}=$

$=-\frac{H_{p}}{R} \frac{\partial}{\partial x} R\left\{\left(\frac{\partial p}{\partial x}\right)+\frac{k r_{c}^{2}}{6}\left(\frac{\partial p}{\partial x}\right)^{3}\right\}$.

When formula (14) is inserted into Eq.(13) the modified Reynolds equation takes the form:

$\frac{1}{R} \frac{\partial}{\partial x} R\left[\left(H^{3}+\frac{3}{2} \phi_{p} r_{c}^{2} H_{p}\right) \frac{\partial p}{\partial x}+\right.$

$\left.+\frac{3 k}{20}\left(H^{5}+\frac{5}{3} \phi_{p} r_{c}^{4} H_{p}\right)\left(\frac{\partial p}{\partial x}\right)^{3}\right]=12 \mu \frac{\partial H}{\partial t}$.

If the film thickness is regarded as a random quantity, a height distribution function must be associated. Many real bearing surfaces show a roughness height distribution which is closely Gaussian, at least up to three standard deviations. From a practical point of view, the Gaussian distribution is rather inconvenient and therefore a polynomial form of its approximation is chosen. Following Christensen (1970; 1971; 1973) such a probability density function is

$f\left(h_{s}\right)=\left\{\begin{array}{lc}\frac{35}{32 c^{7}}\left(c^{2}-h_{s}^{2}\right)^{3}, & -c \leq h_{s} \leq+c \\ 0, & \text { elsewhere }\end{array}\right.$

where $\mathrm{c}$ is the half total range of the random film thickness variable. The function terminates at $c= \pm 3 \sigma$, where $\sigma$ is the standard deviation.

Inserting expected values in Eq.(15) we get the general form of the stochastic Reynolds equation

$\frac{1}{R} \frac{\partial}{\partial x}\left(E\left\{R\left[\left(H^{3}+\frac{3}{2} \phi_{p} r_{c}^{2} H_{p}\right) \frac{\partial p}{\partial x}+\right.\right.\right.$

$\left.\left.\left.+\frac{3 k}{20}\left(H^{5}+\frac{5}{3} \phi_{p} r_{c}^{4} H_{p}\right)\left(\frac{\partial p}{\partial x}\right)^{3}\right]\right\}\right)=12 \mu \frac{\partial E(H)}{\partial t}$

where $\mathrm{E}(\cdot)$ is the expectancy operator defined by

$E(\cdot)=\int_{-c}^{+c}(\cdot) f\left(h_{s}\right) d h_{s}$
The problem is now reduced to devising means of evaluating the left-hand side of Eq.(17) subject to the specific model of roughness.

The calculation of the mean film pressure distribution would require the evaluation of the expected value of various film thickness functions.

The forms of the distribution function described by Eq.(18) are given in (Walicka, 2012).

\section{SOLUTIONS TO THE MODIFIED REYNOLDS EQUATION}

In the present study two types of roughness structure are of interest: the longitudinal (radial) one-dimensional roughness pattern, having the form of long narrow ridges and valleys running in the $x$ direction, and the circumferential (transverse) onedimensional roughness pattern, having the form of long narrow ridges and valleys running in the $\vartheta$ direction (Walicka and Walicki, 2002a; 2002b; Walicka, 2009).

For the longitudinal one-dimensional roughness

$H=h(x, t)+h_{s}(\vartheta, \xi)$

the stochastic Reynolds equation is

$\frac{1}{R} \frac{\partial}{\partial x}\left(R\left\{\left[E\left(H^{3}\right)+\frac{3}{2} \phi_{p} r_{c}^{2} H_{p}\right] \frac{\partial(E p)}{\partial x}+\right.\right.$

$\left.\left.+\frac{3 k}{20}\left[E\left(H^{5}\right)+\frac{5}{3} \phi_{p} r_{c}^{4} H_{p}\right]\left[\frac{\partial(E p)}{\partial x}\right]^{3}\right\}\right)=12 \mu \frac{\partial E(H)}{\partial t}$,

but for the circumferential one-dimensional roughness

$H=h(x, t)+h_{s}(x, \xi)$

the stochastic Reynolds equation is

$\frac{1}{R} \frac{\partial}{\partial x}\left(R\left\{\left[\frac{1}{E\left(H^{-3}\right)}+\frac{3}{2} \phi_{p} r_{c}^{2} H_{p}\right] \frac{\partial(E p)}{\partial x}+\right.\right.$

$\left.\left.+\frac{3 k}{20}\left[\frac{1}{E\left(H^{-5}\right)}+\frac{5}{3} \phi_{p} r_{c}^{4} H_{p}\right]\left[\frac{\partial(E p)}{\partial x}\right]^{3}\right\}\right)=12 \mu \frac{\partial E(H)}{\partial t}$.

Note that it may present both Eqs (20) and (22) in one common form as follows:

$\frac{1}{R} \frac{\partial}{\partial x}\left(R\left\{\left[H_{j}^{(3)}+\frac{3}{2} \phi_{p} r_{c}^{2} H_{p}\right] \frac{\partial(E p)}{\partial x}+\right.\right.$

$\left.\left.+\frac{3 k}{20}\left[H_{j}^{(5)}+\frac{5}{3} \phi_{p} r_{c}^{4} H_{p}\right]\left[\frac{\partial(E p)}{\partial x}\right]^{3}\right\}\right)=12 \mu \frac{\partial E(H)}{\partial t}$,

where

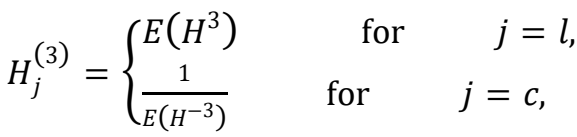

$H_{j}^{(5)}=\left\{\begin{array}{llr}E\left(H^{5}\right) & \text { for } & j=l, \\ \frac{1}{E\left(H^{-5}\right)} & \text { for } & j=c\end{array}\right.$

the case $j=l$ refers to the longitudinal one-dimensional roughness, but the case $j=c-$ to the circumferential one-dimensional roughness.

Consider the case of the Rotem-Shinnar fluid of frequent occurrence for which the factor $k \Lambda_{x y}^{2}<1$; the value of this factor 
indicates that the solutions to the Reynolds equation (23) may be searched in a form of the sum:

$E p=E p^{(0)}+E p^{(1)}$.

Assuming that $E p^{(1)}<<E p^{(0)}$ and substituting Eq.(24) into Eq.(23) we arrive at two linearized equations, the first one

$\frac{1}{R} \frac{\partial}{\partial x}\left\{R\left[H_{j}^{(3)}+\frac{3}{2} \phi_{p} r_{c}^{2} H_{p}\right] \frac{\partial\left(E p^{(0)}\right)}{\partial x}\right\}=12 \mu \frac{\partial E(H)}{\partial t}$,

and the second

$\frac{1}{R} \frac{\partial}{\partial x}\left\{R\left[H_{j}^{(3)}+\frac{3}{2} \phi_{p} r_{c}^{2} H_{p}\right] \frac{\partial\left(E p^{(1)}\right)}{\partial x}\right\}=$

$=-\frac{3 k}{20} \frac{1}{R} \frac{\partial}{\partial x}\left\{R\left[H_{j}^{(5)}+\frac{5}{3} \phi_{p} r_{c}^{4} H_{p}\right]\left[\frac{\partial\left(E p^{(0)}\right)}{\partial x}\right]^{3}\right\}$.

The boundary conditions for pressure are:

- for squeeze film bearing $\left(\frac{\partial H}{\partial t} \neq 0\right)$

$\left.\frac{\partial E p^{(0)}}{\partial x}\right|_{x=0}=0, \quad E p^{(0)}\left(x_{o}\right)=p_{o}$,

$\left.\frac{\partial E p^{(1)}}{\partial x}\right|_{x=0}=E p^{(1)}\left(x_{o}\right)=0$,

- for externally pressurized bearing $\left(\frac{\partial \mathrm{H}}{\partial \mathrm{t}}=0\right)$

$E p^{(0)}\left(x_{i}\right)=p_{i}, \quad E p^{(0)}\left(x_{o}\right)=p_{o^{\prime}}$

$E p^{(1)}\left(x_{i}\right)=E p^{(1)}\left(x_{o}\right)=0$.

The solutions of Eqs (25) and (26) are given, respectively, as follows:

$E p(x, t)=p_{o}-12 \mu\left[F_{o}-F(x, t)\right]$

and

$E p(x)=-\frac{3 k C^{3}}{20} G(x)+\frac{\left[A(x)-A_{o}\right]\left(p_{i}+\frac{3 k C^{3}}{20} G_{i}\right)}{A_{i}-A_{o}}-$

$\frac{\left[A(x)-A_{i}\right]\left(p_{o}+\frac{3 k C^{3}}{20} G_{o}\right)}{A_{i}-A_{o}}$,

where:

$I(x, t)=\int \frac{\int R \frac{\partial E(H)}{\partial t} d x}{R\left[H_{j}^{(3)}+\frac{3}{2} \phi_{p} r_{c}^{2} H_{p}\right]} d x$,

$J(x, t)=\int\left\{\frac{\left[H_{j}^{(5)}+\frac{5}{3} \phi_{p} r_{c}^{4} H_{p}\right]}{R^{3}\left[H_{j}^{(3)}+\frac{3}{2} \phi_{p} r_{c}^{2} H_{p}\right]^{4}}\left[\int R \frac{\partial E(H)}{\partial t} d x\right]^{3}\right\} d x$,

$F(x, t)=I(x, t)-\frac{108 k \mu^{2}}{5} J(x, t), \quad F_{o}=F\left(x_{o}, t\right) ;$

$A(x)=\int \frac{d x}{R\left[H_{j}^{(3)}+\frac{3}{2} \phi_{p} r_{c}^{2} H_{p}\right]}, \quad A_{i}=A\left(x_{i}\right)$,

$A_{o}=A\left(x_{o}\right), \quad C=\frac{p_{i}-p_{o}}{A_{i}-A_{o}}$,

$G(x)=\int \frac{\left[H_{j}^{(5)}+\frac{5}{3} \phi_{p} r_{c}^{4} H_{p}\right] d x}{R^{3}\left[H_{j}^{(3)}+\frac{3}{2} \phi_{p} r_{c}^{2} H_{p}\right]^{4}}$,

$G_{i}=G\left(x_{i}\right), \quad G_{o}=G\left(x_{o}\right)$.

The load-carrying capacity is defined by

$N=2 \pi \int_{0}^{x_{o}}\left(E p-p_{o}\right) R \cos \phi d x$

or

$N=\pi R_{i}^{2} p_{i}+2 \pi \int_{x_{i}}^{x_{o}} E p R \cos \phi d x$ the sense of angle $\phi$ arises from Fig. 2.

The calculation of the mean film pressure distribution would require the calculation of the expected value for various film thickness. For the distribution function given by Eq. (17) we have (Walicka, 2012):

$$
\begin{aligned}
& E(H)=h, \quad E\left(H^{3}\right)=h^{3}\left(1+\frac{1}{3} Y^{2}\right), \\
& E\left(H^{5}\right)=h^{5}\left(1+\frac{10}{9} Y^{2}+\frac{5}{33} Y^{4}\right), \\
& E\left(H^{-3}\right)=\frac{1}{h^{3}}\left[1+\sum_{i=1}^{\infty} \frac{105(n+1) Y^{2 n}}{(2 n+3)(2 n+5)(2 n+7)}\right], \\
& E\left(H^{-5}\right)=\frac{1}{h^{5}}\left[1+\sum_{n=1}^{\infty} \frac{35(n+1)(n+2) Y^{2 n}}{2(2 n+5)(2 n+7)}\right], \quad Y=\frac{c}{h} .
\end{aligned}
$$

\section{RADIAL THRUST BEARING WITH SQUEEZED FILM}

The radial thrust bearing with squeezed film of lubricant is modelled by two parallel disks (Fig. 3).

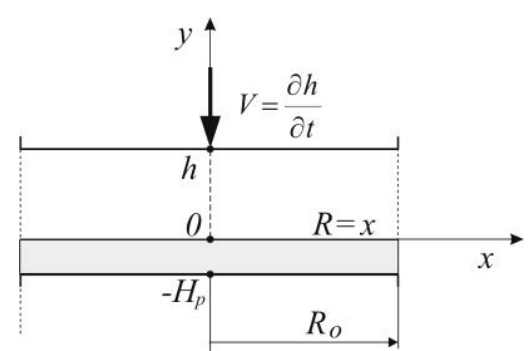

Fig. 3. Squeeze film in a radial thrust bearing

a)

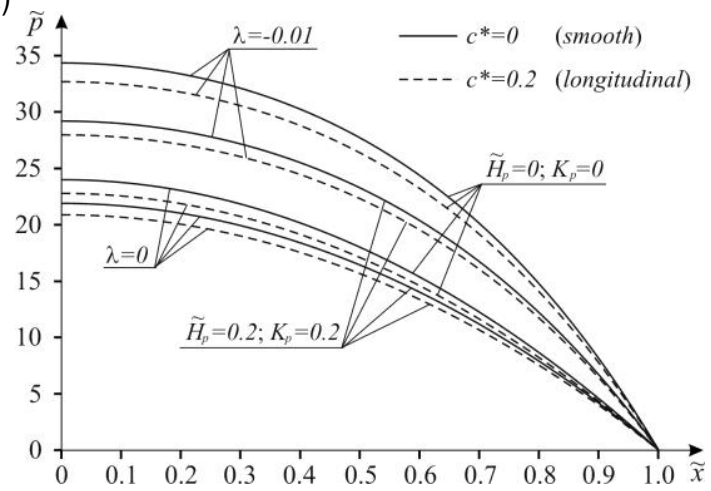

b)

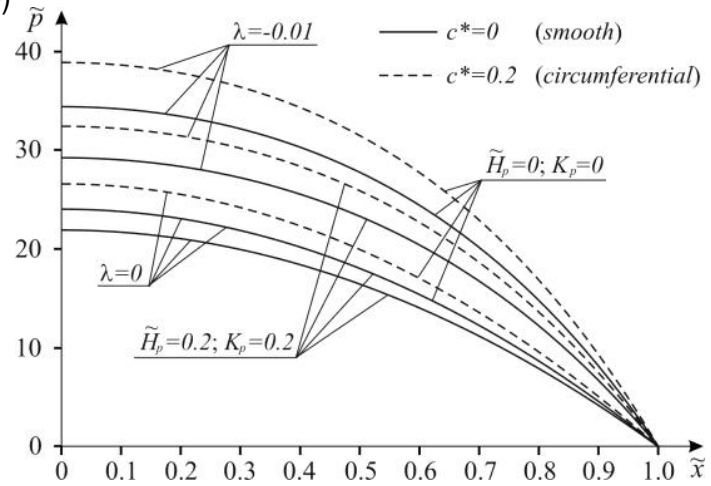

Fig. 4. Dimensionless pressure distribution in the squeeze film thrust bearing with rough surfaces for $\widetilde{H}_{p}=0.2, K_{p}=0.2$, $\lambda=-0.01$ and $\lambda=0$ and $\varepsilon=0.5$; (a) longitudinal roughness and (b) circumferential roughness 
a)

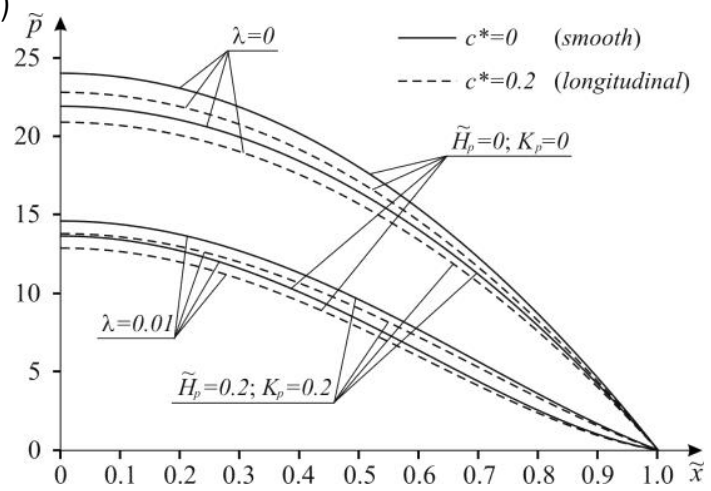

b)

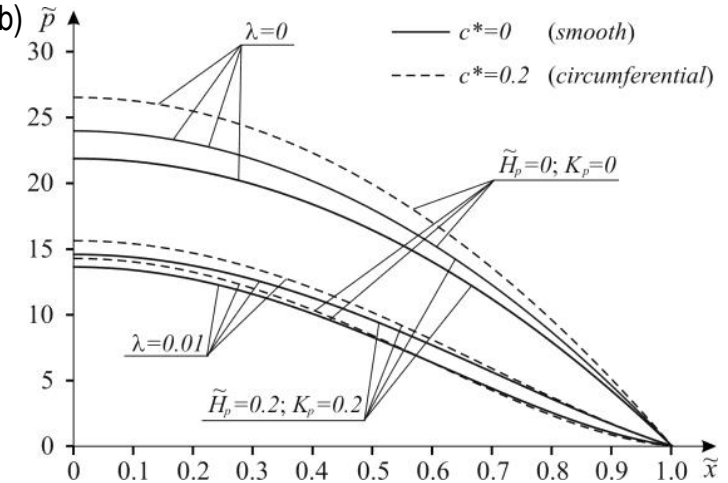

Fig. 5. Dimensionless pressure distribution in the squeeze film thrust bearing with rough surfaces for $\widetilde{H}_{p}=0.2, K_{p}=0.2, \lambda=0$ and $\lambda=0.01$ and $\varepsilon=0.5$; (a) longitudinal roughness and (b) circumferential roughness

a)

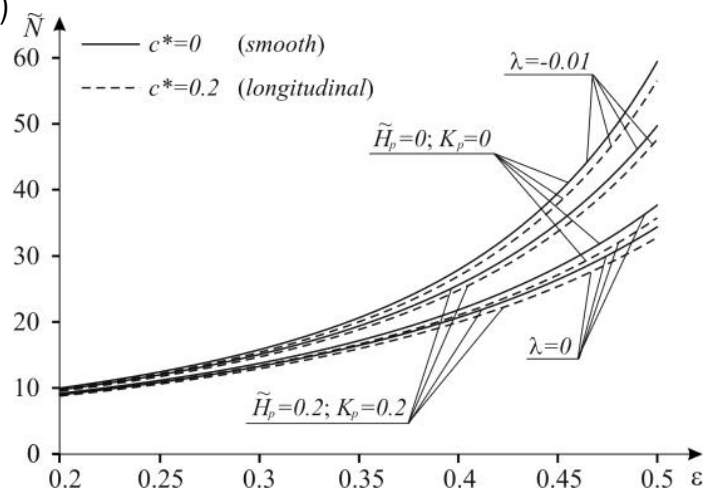

b)

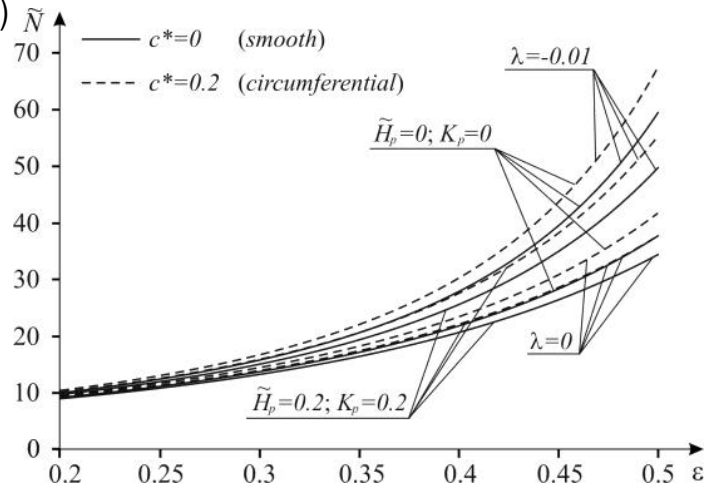

Fig. 6. Load-carrying capacity for the squeeze film thrust bearing with rough surfaces for $\widetilde{H}_{p}=0.2, K_{p}=0.2, \lambda=-0.01$ and $\lambda=0$ and $\varepsilon=0.5$; (a) longitudinal roughness and (b) circumferential roughness
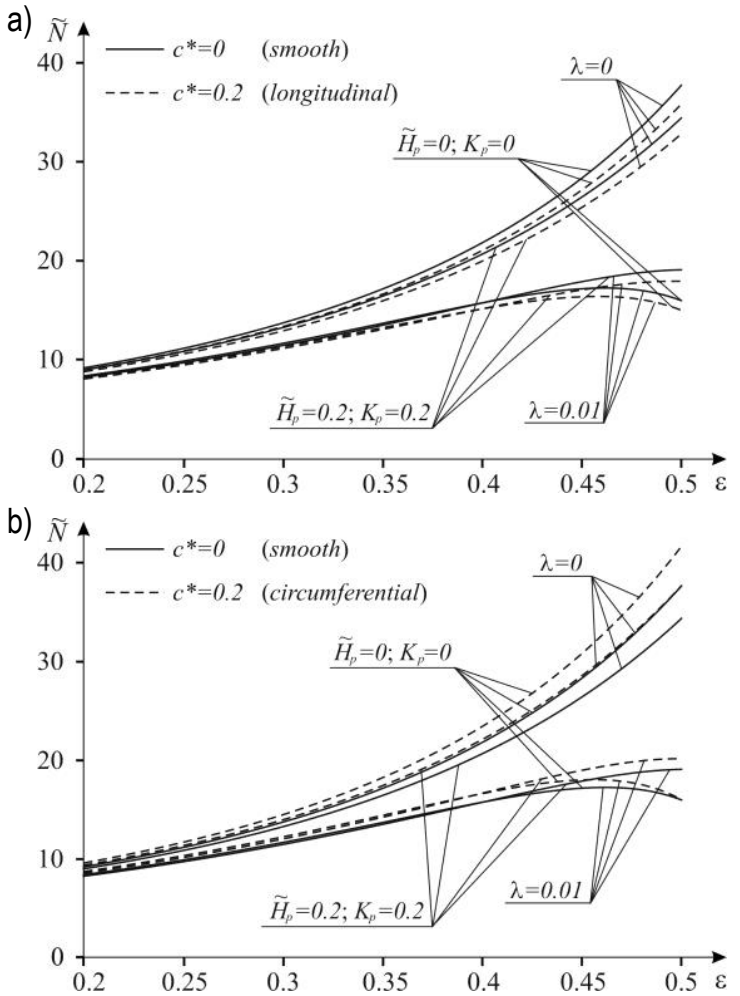

Fig. 7. Load-carrying capacity for the squeeze film thrust bearing with rough surfaces for $\widetilde{H}_{p}=0.2, K_{p}=0.2, \lambda=0$ and $\lambda=0.01$ and $\varepsilon=0.5$; (a) longitudinal roughness and (b) circumferential roughness

Introducing the following parameters:

$$
\begin{aligned}
& \tilde{x}=\frac{x}{R_{o}}, x=R, \quad \tilde{R}=\frac{R}{R_{o}}, \tilde{h}=\frac{h}{h_{o}}=e(t), \\
& e(t)=1-\varepsilon(t), \quad K_{p}=\frac{r_{c}}{h_{o}}, \widetilde{H}_{p}=\frac{\phi_{p} H_{p}}{h_{o}}, \\
& \tilde{p}=\frac{\left(E p-p_{o}\right)}{\mu \dot{\varepsilon}}\left(\frac{h_{o}}{x_{o}}\right)^{2}, \dot{\varepsilon}=\frac{d \varepsilon}{d t}, \widetilde{N}=\frac{N h_{o}^{2}}{\mu \dot{\varepsilon} x_{o}^{4}}, \\
& \lambda=k\left(\frac{\mu \dot{\varepsilon} x_{o}}{h_{o}}\right)^{2}
\end{aligned}
$$

we will obtain the formulae for the dimensionless pressure distribution and load-carrying capacity for the radial thrust bearing with a squeeze film of the Rotem-Shinnar type lubricant:

$\tilde{p}=\frac{3}{M_{j}^{(3)}}\left[1-\tilde{x}^{2}-\frac{27}{10} \lambda \frac{M_{j}^{(5)}}{\left(M_{j}^{(3)}\right)^{3}}\left(1-\tilde{x}^{4}\right)\right]$,

$\widetilde{N}=\frac{3 \pi}{2 M_{j}^{(3)}}\left[1-\frac{18}{5} \lambda \frac{M_{j}^{(5)}}{\left(M_{j}^{(3)}\right)^{3}}\right]$,

where:

$M_{j}^{(3)}=\widetilde{H}_{j}^{(3)}+\frac{3}{2} K_{p}^{2} H_{p}, \quad M_{j}^{(5)}=\widetilde{H}_{j}^{(5)}+\frac{5}{3} K_{p}^{4} H_{p}$,

$c^{*}=\frac{c}{h_{0}}$, 


$$
\begin{gathered}
\widetilde{H}_{j}^{(3)}= \begin{cases}e^{3}\left[1+\frac{1}{3}\left(\frac{c^{*}}{e}\right)^{2}\right] & \text { for } j=l, \\
\left(\frac{1}{e^{3}}\left[1+\frac{2}{3}\left(\frac{c^{*}}{e}\right)^{2}\right]\right)^{-1} & \text { for } j=c,\end{cases} \\
\widetilde{H}_{j}^{(5)}= \begin{cases}e^{5}\left[1+\frac{10}{9}\left(\frac{c^{*}}{e}\right)^{2}\right] & \text { for } j=l, \\
\left(\frac{1}{e^{5}}\left[1+\frac{5}{3}\left(\frac{c^{*}}{e}\right)^{2}\right]\right)^{-1} & \text { for } j=c .\end{cases}
\end{gathered}
$$

\section{CONCLUSIONS}

The modified Reynolds equation for a Rotem-Shinnar type of pseudo-plastic lubricants flowing in a clearance of the thrust curvilinear bearing with rough surfaces is derived; to one bearing surface a porous layer adheres. As a result the general formulae for pressure distributions and load-carrying capacity are obtained.

If follows from carried out calculations and their graphic presentations that the both magnitudes are dependent on the signs of rheological parameters $k$ or $\lambda$.

For squeeze film radial bearings the pressures and loadcarrying capacities increase with a decrease of the $\lambda$ values with respect to the suitable values of Newtonian lubricants.

Basing on the adequate formulae for thrust externally pressurized bearing it may conclude that this phenomenon should run inversely. Note the changes of the bearing parameters presented in this paper for rough surfaces and the Rotem-Shinnar lubricant are similar to those for smooth surfaces (Walicki, 2005). The bearing surfaces porosity, expressed as a product of the parameters $\widetilde{H}_{p}$ and $K_{p}$, results in some small decrease of the pressure and load-carrying capacity.

Nomenclature: $\boldsymbol{A}_{1}$ - the first Rivlin-Ericksen kinematic tensor, $c$ - maximum asperity deviation, $c^{*}$ - nondimensional roughness parameter, $e(t)$ - bearing squeezing, $E(\bullet)$ - expectancy operator, $f\left(h_{s}\right)$ - probability density distribution function, $h(x, t)-$ nominal film thickness, $h_{s}(x, \vartheta, \xi)$ - random deviation of film thickness, $H$ - film thickness, $H_{p}$ - porous pad thickness, $k, k_{i}$ - pseudo-plasticity coefficients, $N$ - load-carrying capacity, $p$ - pressure, $r$ - radius, $R, R(x)$ - local radius of the lower bearing surface, $v_{x}, v_{y}$ - velocity components, $x, y-$ orthogonal coordinate, $\varepsilon(t)$ - squeezing ratio, $\vartheta$ - angular coordinate, $\mu$ - coefficient of viscosity, $\xi$ - random variable, $\rho$ - fluid density.

\section{REFERENCES}

1. Bujurke N.M., Kudenatti R.B., Awati V.B. (2007), Effect of surface roughness on squeeze film poroelastic bearings with special reference to synovial joints, Mathematical Biosciences, 209, 76-89.

2. Christensen H. (1970), Stochastic model for hydrodynamic lubrication of rough surfaces, Proc. Inst. Mech. Engrs, 184, No. 1, 1013-1022.
3. Christensen H., Tønder K. (1971), The hydrodynamic lubrication of rough bearing surfaces of finite width, ASME, J. Lubric. Technol., 93(2), 324-330.

4. Christensen H., Tønder K. (1973), The hydrodynamic lubrication of rough journal bearings, ASME, J. Lubric. Technol., 95(1), 166-172.

5. Dorier C., Tichy J. (1992), Behaviour of a Bingham-like viscous fluid in lubrication flows, J. Non-Newt. Fluid Mech., 45(3), 291-350.

6. Gururajan K., Prakash J. (1999), Surface roughness effects in infinitely long porous journal bearing, Journal of Tribology, Trans. ASME, 121(1), 139-147.

7. Khonsari M.M., Dai F. (1992), On the mixture flow problem in lubrication of hydrodynamic bearing: small solid volume fraction, STLE Trib. Trans., 35(1), 45-52.

8. Lin J.-R. (2000), Surfaces roughness effect on the dynamic stiffness and damping characteristics of compensated hydrostatic thrust bearings, Int. J. Machine Tools Manufact., 40, 1671-1689.

9. Lin J.-R. (2001), The effect of couple stresses in the squeeze film behavior between isotropic rough rectangular plates, Int. J. Appl. Mech. Eng., 6(4), 1007-1024.

10. Lipscomb C.C., Denn M.M. (1984), Flow of Bingham fluids in complex geometries, J. Non-Newt. Fluid Mech., 14(3), 337-349.

11. Morgan V.T., Cameron A. (1957), Mechanismus of lubrication in porous metal bearings, Proc. Conf. on Lubrication and Wear, Inst. Mech. Eng., 151-157, London.

12. Prakash J., Tiwari K. (1984), An analysis of the squeeze film between rough porous rectangular plates with arbitrary porous wall thickness, Journal of Tribology, Trans. ASME, 106(2), 218-222.

13. Prakash J., Tiwari K. (1985), Effects of surface roughness on the squeeze film between rectangular porous annular disc with arbitrary porous wall thickness, Int. J. Mech. Sci., 27(3), 135-144.

14. Rajalingam C., Rao B.V.A., Prabu S. (1978), The effect of a nonNewtonian lubricant on piston ring lubrication, Wear, 50, 47-57.

15. Rotem Z., Shinnar R. (1961), Non-Newtonian flow between parallel boundaries in linear movements, Chem. Eng. Sie., 15, 130-143.

16. Swamy S.T.N., Prabhu B.S., Rao B.V.A. (1975), Stiffness and damping characteristics of finite width journal bearing with a nonNewtonian film and their application to instability prediction, Wear, 32, 379-390.

17. Wada S., Hayashi H. (1971), Hydrodynamic lubrication of journal bearings by pseudo-plastic lubricants, Pt 1, Theoretical studies, Pt 2 , Experimental studies, Bull. JSME, 14(69), 268-286.

18. Walicka A. (1989), Accurate and Asymptotic Solution of Simplified Sets of Equations Describing the Motion of Viscous Fluids in a Slot Bounded by Two Co-axial Surfaces of Revolution (in Polish), PWN, Warszawa.

19. Walicka A. (1994), Micropolar flow in a Slot Between Rotating Surfaces of Revolution, TU Press, Zielona Gora.

20. Walicka A. (2002), Rotational Flows of Rheologically Complex Fluids in Thin Channels (in Russian), University Press, Zielona Gora.

21. Walicka A. (2009), Surface roughness effects in a curvilinear squeeze film bearing lubricated by a power-law fluid, Int. J. Appl. Mech. Engng, 14(1), 277-293.

22. Walicka A. (2012), Porous curvilinear squeeze film bearing with rough surfaces lubricated by a power-law fluid, Journal of Porous Media, 15(1), 29-49.

23. Walicka A., Walicki E. (2002a), Surface roughness effect on the pressure distribution in curvilinear thrust bearings, Exploitation Problems of Machines, 131(3), 157-167.

24. Walicka A., Walicki E. (2002b), Couple stress and surface roughness effects in curvilinear thrust bearings, Int. J. Appl. Mech. Engng, Vol. 7, Spec. Issue: SITC, 109-117.

25. Walicki E. (1975), Viscous fluid flow in a slot of the curvilinear thrust bearing, Rev. Roum. Sci. Techn. - Mech. Appl., 20(4), 483-493. 